\title{
The TRIUMF Annular Chamber of Tracking and Identification of Charged Particles (TACTIC)
}

\author{
G. Ruprecht ${ }^{* b}$, P. Amaudruz ${ }^{b}$, P. Mumby-Croft ${ }^{a}$, L. Buchmann ${ }^{b}$, S. Fox ${ }^{a}$, B. Fulton $^{a}$,

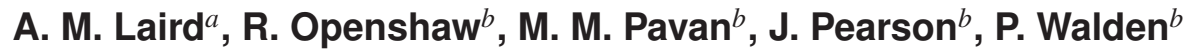 \\ ${ }^{a}$ University of York, U.K. \\ ${ }^{b}$ TRIUMF national laboratory, Canada \\ E-mail: ruprechtetriumf.ca
}

\begin{abstract}
Many nuclear reactions important for nucleosynthesis are investigated by transfer reactions using a radioactive ion beam on gas targets such as ${ }^{1} \mathrm{H},{ }^{2} \mathrm{H},{ }^{3} \mathrm{He}$ and ${ }^{4} \mathrm{He}$. Since these measurements are performed in inverse kinematics, the energies of the ejectiles are usually low with a strong angular dependence in the laboratory system. Therefore, a new kind of detector is needed that allows the detection of low-energy ions from a gas target with good energy and angle resolution and a large angular coverage. TACTIC is a cylindrical time-projection chamber that can utilize the target gas also as the drift medium so that no separation window is required. Moreover, the reaction region is still separated from the drift region which allows for the higher beam currents needed for impact energies far below the Coulomb barrier. The cylindrical design is possible by the application of gas electron-multiplier (GEM) foils for the amplification of the weak electrondrift signals. From the electron- drift times the ion track can be reconstructed while the collected charge provides additional information about the ion species.
\end{abstract}

International Symposium on Nuclear Astrophysics - Nuclei in the Cosmos - IX

June 25-30 2006

CERN, Geneva, Switzerland

${ }^{*}$ Speaker. 


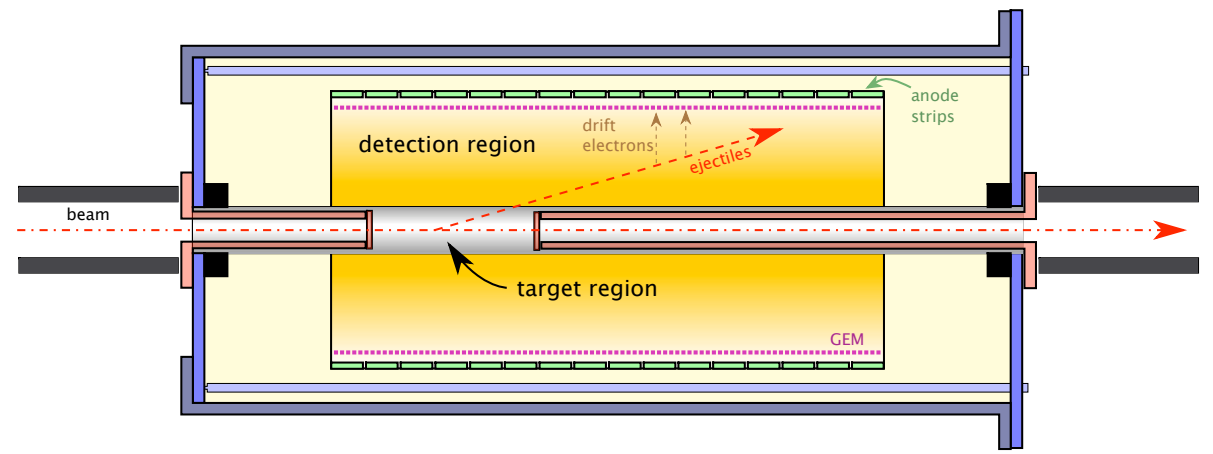

Figure 1: Schematic view of the TACTIC chamber. Wires are stretched along the target tube and biased with a negative high voltage. Another (inner) system of wires suppresses beam induced electrons. The electrical connectors on the right hand side provide the high voltages for the wires and the GEM and lead the signals to the outside. The anode cylinder is divided into 48 strips. The detection cylinder is $24 \mathrm{~cm}$ long and has a diameter of $6 \mathrm{~cm}$.

\section{Chamber design}

The principal design of TACTIC $[1,2]$ is shown in figure 1 . The beam particles coming from the left move into the gas filled target tube, while the ejectiles move into the detection region where they induce secondary electrons. An electric field between the target tube and the anode strips forces them to drift to the gas electron multiplier (GEM) [3, 4] foil where the signal is pre-amplified and then picked up by the anode strips. From the measured drift times the radial coordinates of the particle tracks can be deduced, and from the measured charge the energy loss for each anode strip can be derived. The GEM makes it possible to give the chamber a cylindrical design and, therefore, to separate target and detection region. Since the target region is "blind", higher beam currents can be applied than in other time projection detectors. For many reactions (see e.g. ${ }^{8} \mathrm{Li}(\alpha, \mathrm{n})$ described below) target gas and detection are the same, so that no separation foil is needed and low-energy ejectiles can be detected.

There is a complicated dependence between the reaction kinematics, the detection/target gas pressure, the reaction yield, and the energy loss of the ejectiles (and projectiles). All these dependencies fix the geometry of the chamber. For this reason a simulation has been implemented using CERN's GEANT4 framework. A Coulomb single-scattering process (not yet available in GEANT4) has been programmed and the ion physics has been improved. An example of simulated ion tracks for the ${ }^{8} \mathrm{Li}+{ }^{4} \mathrm{He}$ reaction as detected by the 48 anode pads is shown in fig. 2 .

\section{Gas mixture dependence}

A series of measurements with a planar test chamber has been performed in order to determine the best composition of the detection gas and the performance of the GEM. Fig. 3 (left) shows the operation range of the GEM for a $90 \% \mathrm{He} / 10 \% \mathrm{CO}_{2}$ volume gas mixture at different pressures. The tests show that even at lower pressures (which are necessary for low-energy ejectiles) there is a good amplification. The noise limit will be even lower for the TACTIC prototype, so that a large 


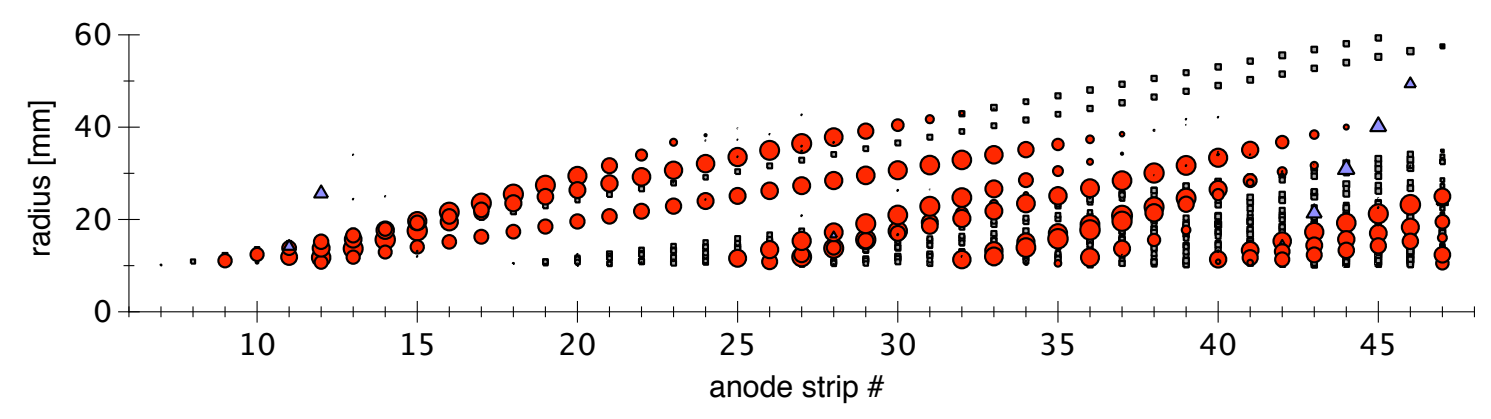

Figure 2: Simulated ion tracks for the ${ }^{8} \mathrm{Li}+{ }^{4} \mathrm{He}$ reaction as seen by the 48 anode pads which can detect only electrons from the drift region $(\mathrm{r}=10-60 \mathrm{~mm})$. Circles $={ }^{11} \mathrm{~B}$, squares $={ }^{8} \mathrm{Li}$, triangles $={ }^{4} \mathrm{He}$. The size of the symbols reflects the energy collected per anode strip.
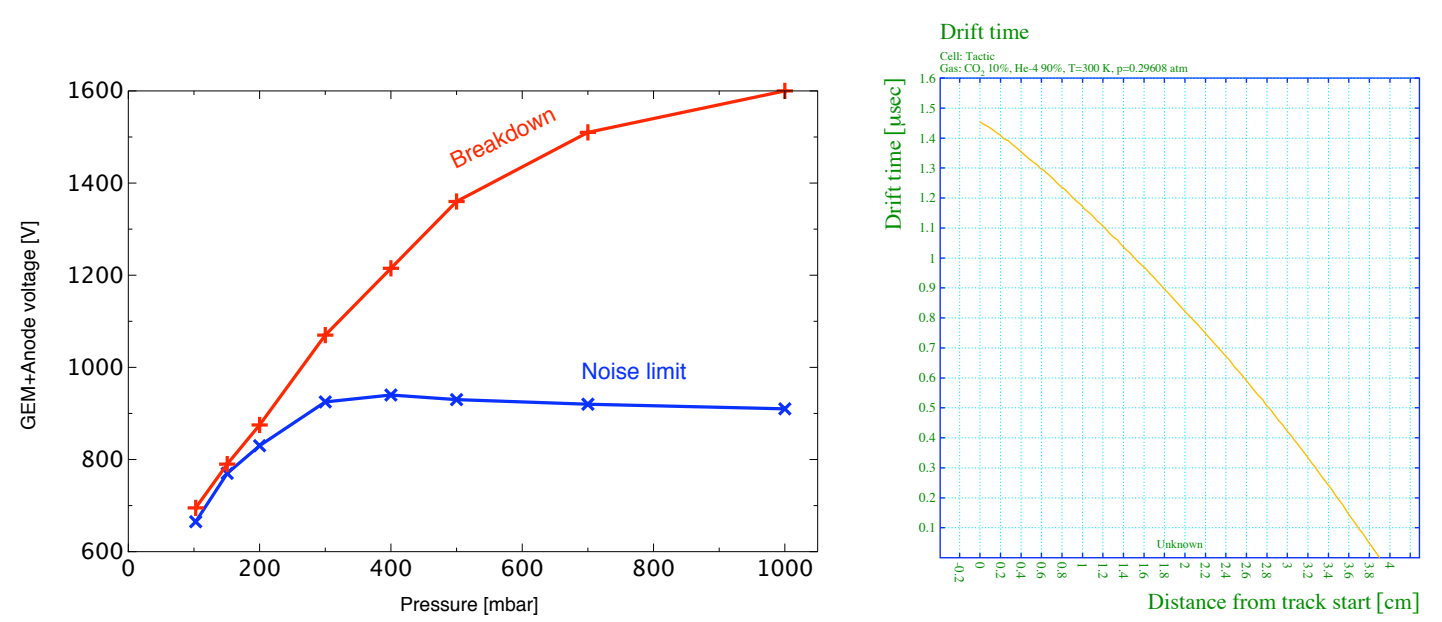

Figure 3: Left: Voltage range in which an amplified signal can be detected vs. gas pressure. Right: GARFILED calculation of the electron drift times.

pressure range is available, sufficient for most astrophysical applications. Fig. 3 (right) shows a GARFIELD [5] calculation of the electron drift times vs. the radial distance in the chamber where they are produced at a pressure of $300 \mathrm{mbar}$ as needed for the ${ }^{8} \mathrm{Li}(\alpha, \mathrm{n})$ reaction. Ionization crosssections from Magboltz [6] have been used for this calculation.The linearity is excellent for this purpose.

\section{First measurements}

The first planned reactions to be measured are ${ }^{8} \operatorname{Li}(\alpha, n){ }^{11} \mathrm{~B}$ and ${ }^{7} \mathrm{Be}+\mathrm{p}$ elastic scattering. They both can be measured with a radioactive beam in inverse kinematics. The low cross-section and the large ejectile cone favour TACTIC for these experiments.

\section{$3.1{ }^{8} \operatorname{Li}(\alpha, \mathbf{n}){ }^{11} B$}

R-process calculations of nucleosynthesis in neutrino driven winds in supernovae also includ- 
ing light elements [7] found two new reaction chains that can change the heavy element synthesis by one order of magnitude. A very large uncertainty in these chains comes from the only poorly known ${ }^{8} \operatorname{Li}(\alpha, \mathrm{n}){ }^{11} \mathrm{~B}$ cross-section. The lowest important temperature of $T_{9}=0.6$ corresponds to a ${ }^{8} \mathrm{Li}$ impact energy of 100-200 keV/u. In previous measurements [8] a time projection chamber was used as well. However, since there was no separation between target and detection region only a limited beam intensity could be applied. At the ISAC/TRIUMF facility, higher ${ }^{8} \mathrm{Li}$ intensities are available at low energies $(120 \mathrm{keV} / \mathrm{u})$, and with TACTIC the available data set could be greatly improved.

\section{2 ${ }^{7} \mathrm{Be}+\mathrm{p}$ elastic scattering}

The famous ${ }^{7} \mathrm{Be}(\mathrm{p}, \gamma)^{8} \mathrm{~B}$ still has uncertainties at low energies mainly arising from conflicting results of direct and Coulomb dissociation measurements (see [9] for a description of the problem). With the high angular range covered by TACTIC, ${ }^{7} \mathrm{Be}(\mathrm{p}, \mathrm{p})$ scattering phase shifts (there are 16 involved) could be measured which contributes to a better understanding of the ${ }^{7} \mathrm{Be}(\mathrm{p}, \gamma){ }^{8} \mathrm{~B}$ reaction. This measurement is more challenging since the very high proton energy requires a heavier drift gas. Therefore, the detection gas is not the same as the target gas and a separation foil surrounding the target region is needed.

The chamber prototype is now being built at the University of York, U.K., and will be tested at the end of this year at TRIUMF with a stable beam.

\section{References}

[1] TACTIC website http://tactic.triumf.ca.

[2] G. Ruprecht, et al., Europ. Phys. J. A 27(2006)315

[3] F. Sauli and A. Sharma, Ann. Rev. Nucl. Part. Sc. 49 (1999) 341

[4] A. Sharma and F. Pauli, Nuc. Inst. Meth. A 350, (1994) 470

[5] R. Veenhof, GARFIELD web site: http://garfield.web.cern.ch

[6] S. Biagi, MAGBOLTZ web site: http://consult.cern.ch/writeup/magboltz/

[7] M. Terasawa, et al., Astrop. J. 562(2001)470

[8] T. Hasimoto, et al., Nuc. Phys. A 764(2004)330

[9] C. A. Bertulani, Phys. Rev. Lett. 94(2005)072701 